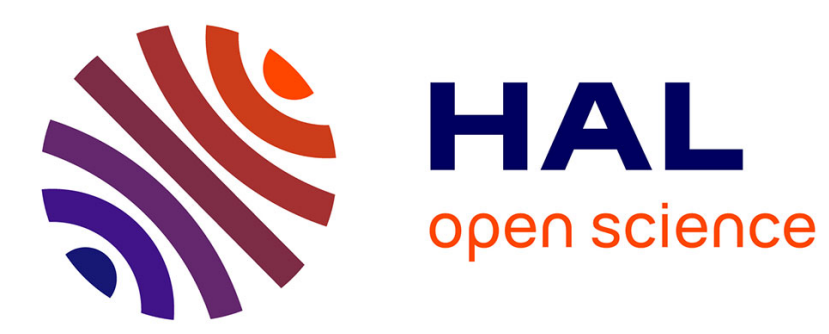

\title{
Inner Speech sustains Predictable Task Switching: Direct Evidence in Adults
}

Lucie Laurent, Jean-Louis Millot, Patrice Andrieu, Valérie Camos, Caroline Floccia, Fabien Mathy

\section{- To cite this version:}

Lucie Laurent, Jean-Louis Millot, Patrice Andrieu, Valérie Camos, Caroline Floccia, et al.. Inner Speech sustains Predictable Task Switching: Direct Evidence in Adults. Journal of Cognitive Psychology, 2016, 28, 10.1080/20445911.2016.1164173 . hal-01371987

\section{HAL Id: hal-01371987 \\ https://hal.science/hal-01371987}

Submitted on 28 Sep 2016

HAL is a multi-disciplinary open access archive for the deposit and dissemination of scientific research documents, whether they are published or not. The documents may come from teaching and research institutions in France or abroad, or from public or private research centers.
L'archive ouverte pluridisciplinaire HAL, est destinée au dépôt et à la diffusion de documents scientifiques de niveau recherche, publiés ou non, émanant des établissements d'enseignement et de recherche français ou étrangers, des laboratoires publics ou privés. 


\title{
Inner Speech sustains Predictable Task Switching:
}

\section{Direct Evidence in Adults}

\author{
Lucie Laurent ${ }^{1,2}$, Jean-Louis Millot ${ }^{3}$, Patrice Andrieu ${ }^{3}$, \\ Valérie Camos $^{4}$, Caroline Floccia ${ }^{5}$, and Fabien Mathy ${ }^{2}$
}

\author{
${ }^{I}$ Maison des Sciences de l'Homme et de l'Environnement Ledoux \\ ${ }^{2}$ Laboratoire de psychologie de Besançon, Université de Franche Comté \\ ${ }^{3}$ Laboratoire de neurosciences de Besançon, Université de Franche Comté \\ ${ }^{4}$ Département de psychologie, Université de Fribourg \\ ${ }^{5}$ School of Psychology, Plymouth University
}

Key Words: task switching, cognitive control, inner speech, language, electromyography. Corresponding Author:

Fabien Mathy. Université Nice Sophia Antipolis, BCL Lab., UMR 7320, Nice, France. fabien.mathy@unice.fr. Tel: 01133489881445. This research was supported in part by a grant from the Région de Franche-Comté AAP2010 awarded to Fabien Mathy and Lucie Laurent 
with the support of the Maison des Sciences de l'Homme et de l'Environnement Ledoux USR 3124, and by funds from the MTC-NSC (Université Nice Sophia Antipolis) awarded to Fabien Mathy. 


\begin{abstract}
It has been proposed that inner speech supports task selection in task switching studies, especially when the need for endogenous control is increased. This has been established through the suppression of inner speech in cognitive-flexibility tasks that leads to poorer performance. The aim of this study is to quantify the role of inner speech in a flexibility task by using surface laryngeal electromyography, which, contrary to previous studies, enables participants to freely verbalize the tasks. We manipulated endogenous and exogenous flexibility in a mathematical switching task paradigm. Experiment 1 shows that inner speech acts as a support for switching and is recruited more often when the tasks are of an endogenous type. The main result of Experiment 2 that language is recruited more for the mixing cost than for the switch cost (regardless of the endogenous factor) extends past findings obtained through articulatory suppression.
\end{abstract}




\section{Inner Speech sustains Predictable Task Switching: Direct Evidence in Adults}

Although several models have been developed to account for switching processes (e.g., Altmann \& Gray, 2008; Gilbert \& Shallice, 2002; Meiran, 2000), the specific role of speech in switching and more specifically, inner speech, remains to be clarified. Inner speech has long been hypothesized to be a vehicle of thought and voluntary control (e.g., Luria, 1969; Reed, 1916), and one of the most common techniques used to explore the role of inner speech in flexibility tasks is to disrupt speech through the use of articulatory suppression (Baddeley, Chincotta, \& Adlam, 2001; Bryck \& Mayr, 2008; Cinan \& Tanor, 2002; Dunbar \& Sussman, 1995; Emerson \& Miyake, 2003; Goschke, 2000; Kray, Eber, \& Lindenberger, 2004; Miyake, Emerson, Padilla, \& Ahn, 2004; Saeki \& Saito, 2004). From these studies inner speech appears to be an important determinant of task switching. For example, the switch cost (i.e., the difference in RT between task-switch trials and consecutive same-task trials) was found to increase when subjects were unable to use inner speech (e.g. Emerson \& Miyake, 2003). One interpretation is that inner speech contributes to effectively recode tasks and facilitates executive processes, a language function sometimes referred to as extracommunicative (Lupyan, 2009).

Previous studies have shown that verbal self-instructions can be used to support task switching (Kirkham, Breeze, \& Marí-Beffa, 2012; Kray, Eber, \& Karbach, 2008), suggesting that encouraging the use of speech rather than denying it can have beneficial effects. Following up on these findings we decided to promote its use and directly quantify its involvement by adding laryngeal surface electromyography measurements (EMG) to behavioral parameters such as response time and switch cost. One advantage of this method is to avoid using articulatory suppression, which can be viewed by participants as a non-natural process that disrupts task performance. The EMG device, which measures the electrical impulses of muscles using electrodes applied to the surface of the skin, makes it possible to 
observe speech signals even when speech is internalized (Garrity, 1977; Sokolov, 1972). The electrical activity of laryngeal muscles can be detected whether or not the face or mouth is moving, and whether or not sound is emitted (Betts, Binsted, \& Jorgensen, 2006; Jou, Schultz, Walliczek, Kraft, \& Waibel, 2006). The current view on the relationship between inner speech and motor activation is that the recruitment of the motor system will depend on the level of inner speech in the task at hand (e.g. Geva et al., 2011; Perrone-Bertolotti, Rapin, Lachaux, Baciu \& Lœvenbruck, 2014), with time-constrained and/or attention-demanding tasks more likely to recruit the motor system. Since the switching tasks used in the current study are both rapid and attention-demanding, we expect participants to fully engage in the motor production system underlying inner speech, allowing us to detect inner speech production with the EMG technique.

To examine how the use of inner speech varies as a function of switching conditions, participants were administered a predictable mathematical switching task that included both cued and non cued trials to directly contrast the effects of exogenous and endogenous control on inner speech in a within-subject design. Endogenous control is required when there is no available external cue that signals the next task to be performed, for instance when one has to remember an alternation pattern to switch from one task to another ( $\mathrm{BBAB}$, etc), while exogenous control is made possible by cuing the upcoming task (for instance, using an arithmetic symbol to indicate which operation to be performed next). Having to memorize a sequence to carry out tasks ( $\mathrm{ABAB}$, etc.) as in endogenous tasks usually slows down the alternation of the tasks in comparison to exogenous tasks because the participant is required to update the alternation process on the fly. In contrast, the presence of external cues as in exogenous tasks is supposed to alleviate the need for internal-self cuing, which has been hypothesized to be associated with the presence of covert self-instructions (Emerson \& 
Miyake, 2003; Miyake, Emerson, Padilla, \& Ahn, 2004). If task-switching costs reflect in part the amount of inner speech produced to recall which task needs to be performed next, we predict a greater reliance on inner speech in endogenous tasks than in exogenous tasks.

Although it is recommended to use different task-switching paradigms to clearly determine the role of verbal strategies in task-switching designs (Kirkham, Breeze, \& Marí-Beffa, 2012), in our first experiment we chose to rely on a previous study by Emerson and Miyake (2003) that evaluated the impact of articulatory suppression in task-switching using list design (AAAA..., BBBB..., and ABAB...). However, instead of disrupting speech, we quantified the quantity of inner speech produced during the response delays. A specific hypothesis was that more covert vocalizations measured by electrophysiological measures can be found when longer response times are expected, and particularly in the non-cued conditions of the mixed blocks in which switches are required. Because switch costs were confounded with mixed costs in the first experiment, a second experiment tested an AABB mixed list design to better examine the relative effects of mixed costs and switch costs when the participants alternated between the tasks without having to switch tasks systematically.

\section{Experiment 1}

The current experimental design is similar to that of Emerson and Miyake (2003) in which the participants had to alternate between additions and subtractions in a mathematical taskswitching paradigm, with cued (exogenous) and non-cued (endogenous) conditions. They showed that disrupting inner speech particularly increased switch costs, and specifically so in the absence of cues. However, instead of disrupting speech via articulatory suppression to show its disruptive effect on switch costs (Emerson \& Miyake, 2003), laryngeal EMG recordings were used in the present experiment to detect motor activity related to the use of inner speech. Our first goal was to quantify the amount of inner speech generated during the 
response time period, and to examine how it would vary as a function of the difficulty of the task (exogenous vs. endogenous) and the degree of switching involved (repeated vs. alternated). Our second and central aim was to examine whether the amount of inner speech as measured by the EMG would match the pattern of response times (RTs). In other words, if inner speech scaffolds the cognitive processes engaged in this time-pressured and attentiondemanding tasks, we expected a direct correspondence between the amount of inner speech and response times.

\section{Participants}

Thirty-two psychology students at the University of Franche-Comté ( $M=22.2$ years; $S D=2.9 ; 10$ males and 22 females) volunteered to participate in this study in exchange for course credits.

\section{Procedure}

The mathematical task-switching paradigm used two conditions: an endogenous flexibility condition (referred to as Non-Cued; no cue was provided, which required the participant to keep track of the alternation of the tasks) and an exogenous flexibility condition (referred to as Cued; the symbols + and - were used to indicate the next task). Instead of the paper-and-pencil version of the original authors, we used a computerized version of the task to allow synchronization with EMG recordings and precise RT measurements. Another main difference with the original study was the use of symbol cues only (instead of using both color cues and symbol cues as in Emerson \& Miyake, 2003), because the symbol cues more strongly reduced the effect of articulatory suppression. All participants were tested in the two conditions, Cued and Non-Cued and the total duration of the experiment was about 20 minutes, including instructions and breaks. Each of the conditions included three blocks 
(Figure 1), one block in which the participants had to subtract the number 3 from each number, one block in which they had to add 3, and a third block in which they had to alternate between addition and subtraction (type of alternation ABABAB). Each condition consisted of a series of 84 two-digit numbers (13-96) with 28 numbers randomly assigned without repetition to each of the three blocks Addition, Subtraction and Alternation, so that no number appeared twice within the same condition. In all, each participant did 2 conditions $\times 3$ blocks $=6$ blocks, which represented a total of 6 blocks $\times 28$ trials $=168$ trials. The order of the first two blocks (one block of subtractions followed by a block of additions) was counterbalanced between the participants, but the third block was systematically the Alternation block across participants. The order of the two conditions (Cued and Non-Cued ) was also counterbalanced across participants. In the Cued condition, the mathematical symbols + or - appeared to the right of each number, whereas no symbol were presented in the Non-cued condition.

\begin{tabular}{|c|c|c|}
\hline & NON CUED & \\
\hline $\begin{array}{c}1^{\text {st }} \text { block: } \\
\text { Add } 3\end{array}$ & $\begin{array}{l}2^{\text {nd }} \text { block: } \\
\text { Subtract } 3\end{array}$ & $\begin{array}{l}3^{\text {rd }} \text { block: } \\
\text { Alternate btw } \\
\quad+3 \&-3\end{array}$ \\
\hline $15=>18$ & $71=>68$ & $29=>32$ \\
\hline $26=>29$ & $67=>64$ & $81=>78$ \\
\hline $47=>50$ & $55=>52$ & $22=>25$ \\
\hline $34=>37$ & $42=>39$ & $13=>10$ \\
\hline Etc. & Etc. & Etc. \\
\hline
\end{tabular}

\begin{tabular}{|ccc|}
\hline & CUED & \\
$\begin{array}{c}1^{\text {st }} \text { block: } \\
\text { Add 3 }\end{array}$ & $\begin{array}{c}2^{\text {nd }} \text { block: } \\
\text { Subtract 3 }\end{array}$ & $\begin{array}{c}3^{\text {rd }} \text { block: } \\
\text { Alternate btw } \\
+3 \text { \& }-3\end{array}$ \\
$15+=>18$ & $71-=>68$ & $29+=>32$ \\
$26+=>29$ & $67-=>64$ & $81-=>78$ \\
$47+=>50$ & $55-=>52$ & $22+=>25$ \\
$34+=>37$ & $42-=>39$ & $13-=>10$ \\
Etc. & Etc. & Etc.
\end{tabular}

Figure 1. Design of the two conditions in the switching task. Note that the $=>$ symbol indicates the expected answer (i.e., the green number on the right side), but this symbol was not displayed during the tasks.

The general instructions to add or subtract 3 to the stimuli, or to alternate in a given order, were presented visually before each block. During the task, each number appeared in the center of the screen and a response box was located in the lower part of the screen. In 
order to answer, the participants had to use a numeric keyboard and had no possibility to correct their answer. Each answer consisted of two digits and the next number appeared immediately after the second digit was typed. The participants were given a two-minute break between the two tasks.

\section{Behavioural and electrophysiological measures}

The E-Prime software that controlled stimulus presentation and RTs collection was synchronized with an electromyograph (EMG $100 \mathrm{C}$, Biopac $\left.{ }^{\circledR}\right)$ monitored by the AcqKnowledge 4.1 software. The electrodes used in this experiment (EL503, Biopac $\left.{ }^{\circledR}\right)$ were silver chloride $(\mathrm{Ag}-\mathrm{AgCl})$ reference electrodes, measuring one centimeter in diameter $(3.5 \mathrm{~cm}$ including the adhesive skin surface). Two pairs of electrodes were positioned one above the other with $4-\mathrm{cm}$ vertical and horizontal gaps $(2 \mathrm{~cm}$ on either side of the vertical medial line) and placed on the laryngeal muscles in order to record voice signals. The ground electrode was attached to the right wrist of each participant. Participants were instructed to refrain from coughing and to avoid the use of covert speech during the tasks.

To analyze the EMG data (first sampled at $1000 \mathrm{~Hz}$ ), we calculated the integral of the speech signals recorded during each stimulus-response interval. First, comb band stop filtering (frequency $=50 \mathrm{~Hz}$ ) was used to process the EMG data in order to remove power line noise from the raw signal data recorded during the entire experiment for one participant, and a root mean square (RMS) function fully corrected the signal (using a time interval of 30 ms) after removing the baseline that was computed across the entire task. After integrating the two RMS-EMG signals using the trapezoidal rule, we averaged our data from the different electrodes to obtain a single integrated EMG value. We then used this signal to reflect the quantity of inner speech produced. In other words, we defined the quantity of speech (QL) as the amount of EMG signal during a given period, obtained by integrating the signal 
(expressed in mV.ms). QL was used to indicate both the intensity and the duration of inner speech. The mean amplitude (expressed in $\mathrm{mV}$ ) during a given period was computed by dividing the integral by the time interval.

In parallel to electromyography we also recorded RTs in milliseconds (excluding incorrect responses from our analyses). Following the terminology used by Meiran (2000, p. 236), we computed alternation costs, which are based on the difference between single task RT (i.e., participants repeat the same task before the mixing block occurs; e.g., AAAA...) and switch task RT (i.e., participants switch between two tasks during the alternating block; e.g., $\mathrm{AB})$. In addition to the analysis of RTs and alternation costs as in Emerson and Miyake (2003), we also report the quantity of inner speech produced during the response delays across the different blocks and conditions. ANOVAs were conducted for each dependent variable (RT, alternation cost, QL integrals and QL amplitudes), with Cues (Cued vs. Noncued) and Block (Repeated task versus Alternated task blocks) as within factors.

\section{Results and Discussion}

Trials with RTs diverting more than 3 standard deviations from the individual mean RT or below $200 \mathrm{~ms}$ were considered outliers and were excluded from the analysis (following Goschke, 2000, p. 337), which corresponded to $1.6 \%$ of the data.

Following Emerson and Miyake's results, we expected longer RTs in the Non-cued condition as compared to the Cued condition, as well as longer RTs in Alternated task blocks than in Repeated task blocks. We also expected larger switch costs overall in the Non-cued condition than in the Cued condition. ANOVA on response times (Figure 2A) showed a significant effect of the factor Block, $F(1,31)=98, p<.001, \eta_{p}{ }^{2}=.76$, due to longer response times in the Alternated task blocks than in the Repeated task blocks. There was no main effect of the factor Cues, $F(1,31)=.8, p=.40$. Although descriptively the non-cued condition led to 
longer RTs than the cued condition for the alternated task block and not in the repeated task block, the Cues $\times$ Block interaction was no significant ${ }^{1}, F(1,31)=1.6, p=.22$. Note that significance was however reached when the extreme values were not removed prior to analyses $\left(F(1,31)=4.7, p=.04, \eta_{p}^{2}=.13\right)$, as this was done in the study by Emerson and Miyake.

Interestingly, when similar analyses were performed on the mean integral of the EMG signal during the RT interval, this interaction effect was significant (Figure 2B). Indeed, an ANOVA on the mean integral of the EMG showed a significant effect of the factor Block, $F(1,31)=44.5, p<.001, \eta_{p}{ }^{2}=.59$, due to larger integrals in the Alternated task condition than in the Repeated task condition. Again, there was no main effect of the factor Cues, $F(1,31)=$ $.6, p=.43$, but this time we observed a significant interaction between the Cues and Blocks, $F(1,31)=4.5, p=.04, \eta_{p}{ }^{2}=.13$, due to a larger difference between the Cued vs. Non-cued conditions in the Alternated task blocks only. This interaction represents the slopes of Figure $2 \mathrm{~B}$, with significant higher switching costs in the Non-cued condition $(M=2.20 \mathrm{mV} . \mathrm{ms} ; S D$ $=2.16)$ than in the Cued condition $(M=1.07 \mathrm{mV} . \mathrm{ms} ; S D=1.90)$.

\footnotetext{
${ }^{1}$ The RT measures cannot however be easily compared because completion times were recorded by the experimenter with a stopwatch in this previous study.
} 

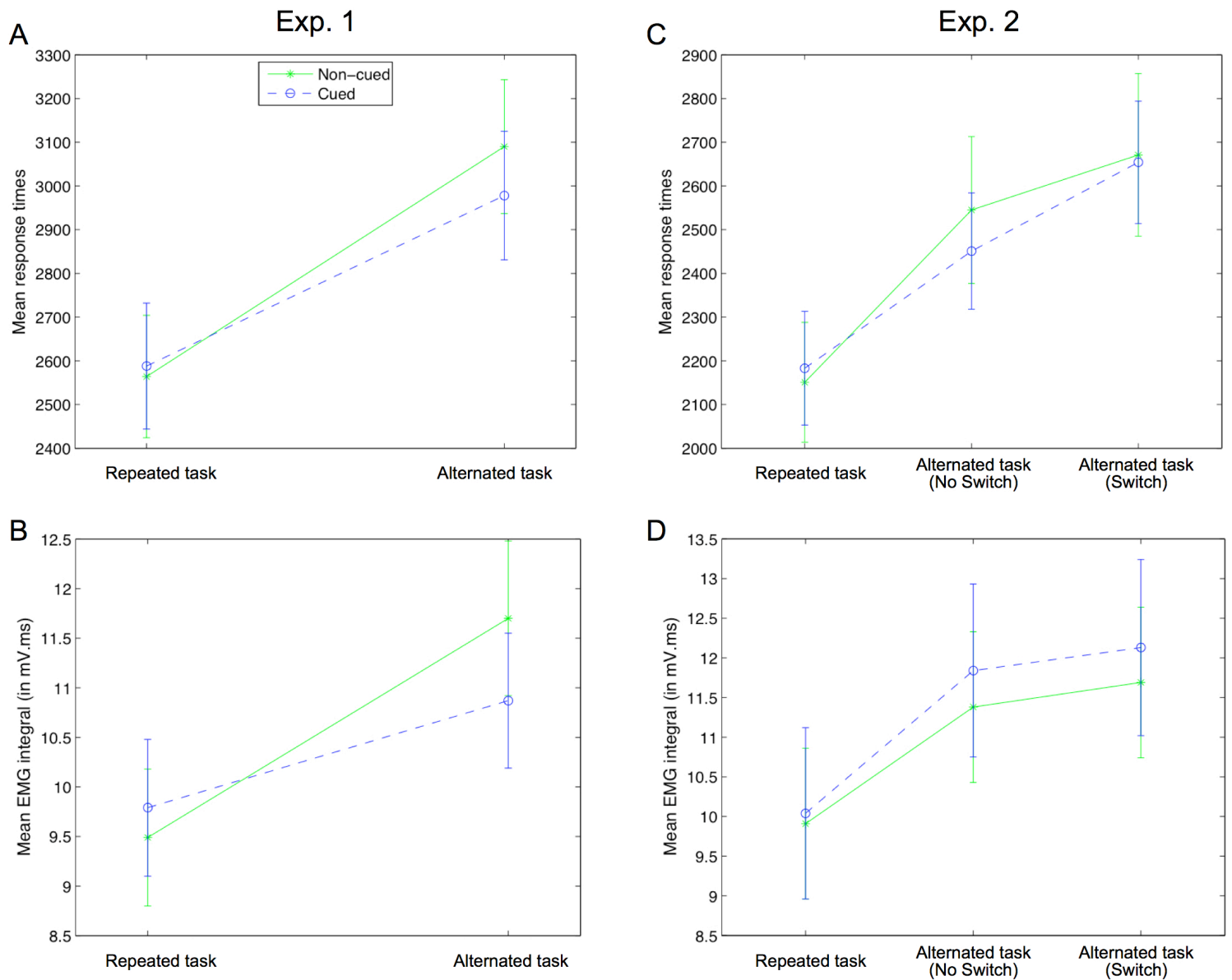

Figure 2. Mean response times and mean EMG integral in Exp. 1 (A, B) and Exp. 2 (C, D) as a function of switch type (Cued vs Non-cued) in the Repeated vs. Alternated task blocks. Exp. 2 further distinguishes Switch trials and No Switch trials.

Note. Error bars are +/- oneSE.

One issue with the current experiment is that switch costs are confounded with mixed costs because the tasks alternated systematically (Bryck \& Mayr, 2005; Lien, Ruthruff, \& Kuhns, 2008; Monsell, 2003), meaning that the differences in EMG between the repeated and the alternated tasks cannot be attributed solely to the cost of switching. This is particularly the case when the next task is not cued. A solution adopted in the next experiment was to use a mixed-task in which a task was either repeated from the previous trial or changed.

\section{Experiment 2}

To better characterize the effect of alternating tasks on inner speech, Experiment 2 was carried out to divide the alternation cost into a mixed cost and a switch cost (Meiran, 2000). 
When participants alternate using an $\mathrm{ABAB}$ pattern, it is impossible to tell whether a longer RT reflects a difficulty to operate the new task (switch cost) or a difficulty to maintain the pattern of task rules (mixed cost), or both. When participants are subjected to an AABB pattern, a same-task transition (hereafter called the no-switch condition) only involves a mixed cost, while a different-task transition (hereafter called a switch condition) implies both a mixed cost and a switch cost .

\section{Participants}

Twenty-three psychology graduate students at the University Nice Sophia-Antipolis $(M=24.5$ years; $S D=5.2 ; 9$ males) volunteered to participate in this study. The experiment was run with a total of 30 participants, but due to technical issues, data for seven participants were corrupted and had to be dismissed.

\section{Procedure}

The experimental design was similar to Experiment 1, except that an AABB pattern of alternating runs of two tasks was used instead of the $\mathrm{ABAB}$ pattern when participants alternated.

\section{Results and Discussion}

As in Experiment 1, trials with RTs diverting more than 3 standard deviations from the individual mean RT or below $200 \mathrm{~ms}$ were considered outliers and were excluded from the analysis, which corresponded to $5.3 \%$ of the data. For each participant, we computed the mean integral EMG and the mean RT, as a function of the three task conditions: Single task, No-switch during the alternation block (this refers to the AA or BB cases) and Switch during the alternation block (referring to either of the $\mathrm{AB}$ and $\mathrm{BA}$ cases), referring later to the Task factor. Then, Mixed costs were computed by subtracting No-switch to Single, and Switch costs were computed by subtracting Switch to No-switch. 
We performed for the two dependent variables (RT, integral EMG) separate repeatedmeasures ANOVAs, which showed a systematic main effect of the three task conditions $\left(F s(2,44)>19, p s<.001 ; \eta_{p}{ }^{2}(\mathrm{RT})=.53, \eta_{p}{ }^{2}(\mathrm{EMG})=.47\right)$, but no effect of the factor Cues and no interaction (see Fig. 2C, D). The respective average RTs for the three conditions (Single task, No-switch and Switch) were $2167 \mathrm{~ms}(S D=130), 2498(140)$, and 2662 (156), with pairwise Bonferroni comparisons showing a significant difference between the Single task condition and the two other conditions of the Task factor. The respective average integral for EMG for the three conditions were 10.0 mV.ms $(S D=1.00), 11.6(.99)$, and $11.9(1.00)$, with pairwise significant differences similar to what was observed in RTs.

Another two separate repeated-measures ANOVAs were run using the factor Cues and the factor Cost (Mixed vs. Switch cost; effects are visible on the two slopes between the three conditions on the $\mathrm{x}$ axis of Fig. 2C, D). We did not reach any significant effect with RTs, but analysis of the EMG signal showed more power. The ANOVA was significant for the factor Cost $\left(F(1,22)=4.7, p=.04 ; \eta_{p}^{2}=.18\right.$, due to a large difference between the integral for the No-Switch condition $(M=1.64, S E=.30)$ and the Switch condition $(M=.30, S E=.39)$. There was no other significant effect, including when testing simple effects. This result tends to indicate that language is recruited more for maintaining the task-set $\mathrm{AABB}$ or updating the next task (A or $\mathrm{B})$ than for switching ( $\mathrm{AB}$ or $\mathrm{BA}$ ) per se (the General Discussion is more specific about whether this large mixed cost on verbalization can be related to a task preparation effect that is present for both repeat and switch trials or switch trials only). 


\section{General Discussion}

The first aim of this study was to establish whether the use of inner speech could be detected online during the execution of switching tasks. Our results clearly demonstrate that participants generate inner speech in these situations, confirming the proposal that the recruitment of the motor system for the production of inner speech is likely to be observed with attention-demanding tasks as those used in the current study (e.g. Geva et al., 2011).

Our second goal was to investigate alternation costs (comparing Repeated task blocks and Alternated task blocks) as a function of the availability of cues (endogenous versus exogenous conditions), and test whether typical RT effects as obtained for example by Emerson and Miyake (2003) would be similarly found in the inner speech measurement patterns. First, we showed that in the absence of an external cue, that is, in the endogenous condition, participants in Exp. 1 presented a slight increase in the production of inner speech as compared to the exogenous condition, showing that they resorted more to inner speech when alternating between the tasks. Figures $2 \mathrm{~A}$ and $2 \mathrm{~B}$ and the statistical outcomes clearly illustrate the close correspondence between response delays in the different conditions and the amount of inner speech generated during these periods. Our result fits nicely to the ideas suggested by Mayr, Kleffner, Kikumoto, and Redford (2014) that speech onset latencies and response times are highly synchronized when performing task sequences, probably because aloud verbalization helped with sequence updating.

Interestingly, the factor Cue did not have a strong effect in Exp. 1, particularly on RTs, regardless of our small sample size, and a similar result was obtained in Exp. 2. Participant debriefing suggest that they felt able to plan the tasks efficiently regardless of the presence of cues, and that because their planning was often taking place ahead of the cues, these cues turned out to be sometimes counter-productive. Therefore, cued or not cued, our tasks were predictable, and this makes it difficult to conclude exactly to what extent the recruitment of 
inner speech in the cued condition relative to the non-cued condition was genuinely due to the recall of the task needed to be performed next. Further experiments need to provide much stronger evidence that inner speech is recruited more often when the tasks are of an endogenous type, especially because we did not find an effect of the endogenous/exogenous factor for the second experiment in our EMG measures. However, one main result in Exp. 2 tends to show that verbalization was more critical for maintaining the task rules AABB or updating the next task A or B (rather than for the actual switching processes $A B$ or $B A$ ), because inner speech was recruited more for mixed costs than for switch costs. Indeed, the slope in Fig. 2 D was steeper between the Repeated task and the Alternated task No-Switch conditions than between the No-switch and Switch conditions of the Alternated tasks. This result fits with the conclusion of Mayr et al. (2014) that inner speech may be recruited as a tool for retrieving and activating the relevant task goal. However, preparing for the tasks at hand is a complex process that has generated some debate over whether the mixing cost is simply reflecting the ability to maintain instructions for all current tasks or the ability to both maintain instructions and to activate the appropriate task for the next trial (see Kieffaber, Kruschke, Cho, Walker, \& Hetrick, 2013, p. 701). Some models assume that there is no need to activate the next task when the task is repeated.

The conclusion we favor, which is that verbalization is more critical for maintaining and updating the task rules, assumes that the preparation process is present for both no-switch conditions (when the trial $n+1$ involves the same task as trial $n$ ) and switch conditions (when the trial $n+1$ involves a different task) of the mixed tasks. Effectively, following Meiran (2000) - and we concur in our analysis - the mixed-cost is computed from substracting the No-switch conditions from the Single task conditions, and computation of the alternation cost subsequently assumes that the preceding mixed-cost (deducted in that case) combines with an 
extra switch cost when the task is being switched. Our analysis did not consider that the mixed-cost could be of a different nature when associated with a switch cost.

Also, the mixed-cost effect was larger on verbalization than on response times: participants took longer to initiate a task switch, but they benefited only minimally from taskrepetition. In addition, this effect was stronger when measuring the inner speech signal than for the response times (the slope in Fig. 2 D was lower than in Fig. 2 C, between the Noswitch and Switch conditions; conversely, the slope was steeper between the Repeated task and the Alternated task No-Switch conditions). Whether related to the repeated trials only or to both repeated trials and switch trials (Kieffaber et al., 2013), our results shows that proactive control seems to generate more verbalization to maintain and update tasks than when specifically switching to a different task. This dissociation between RT and EMG measures seems to indicate that EMG measures provide a better way of quantifying inner speech in cognitive processes compared to response times. However to stay on the safe side it would be more cautious to conclude that electromyography analysis seems more precise than RTs but that feature extraction methods need to be developed to decompose the signal into different components and reduce the noise to support such a strong conclusion.

At this point, these findings confirm that surface electromyography provides a means of quantifying inner speech in cognitive-flexibility processes, in conjunction with more traditional behavioral variables such as response time. Previous studies on the use of inner speech have only gone as far as showing that the suppression of inner speech leads to a decline in task-switching performance. Our study adds to these results by establishing a more direct relationship between decision times and inner speech without disrupting task performance, with results indicating that inner speech might play a predominant role when more endogenous control is required for the task at hand, but mostly when maintaining or updating is required. 


\section{REFERENCES}

Altmann, E. M., \& Gray, W. D. (2008). An integrated model of cognitive control in task switching. Psychological Review, 115(3), 602-639.

Betts, B. J., Binsted, K., \& Jorgensen, C. (2006). Small-vocabulary speech recognition using surface electromyography. Interacting with Computers, 18(6), 1242-1259.

Bryck, R.L. \& Mayr, U. (2005). Does executive task management require verbalization? Memory \& Cognition, 33, 11-23.

Bryck, R. L., \& Mayr, U. (2008). Task selection cost asymmetry without task switching. Psychonomic Bulletin \& Review, 15(1), 128-134.

Dunbar, K., \& Sussman, D. (1995). Toward a cognitive account of frontal lobe function: Simulating frontal lobe deficits in normal subjects. Annals of the New York Academy of Sciences, 769, 289-304.

Emerson, M. J., \& Miyake, A. (2003). The role of inner speech in task switching: A dual-task investigation. Journal of Memory and Language, 48(1), 148-168.

Garrity, L. I. (1977). Electromyography: A review of the current status of subvocal speech research. Memory \& Cognition, 5(6), 615-622.

Gilbert, S. J., \& Shallice, T. (2002). Task switching: A PDP model. Cognitive Psychology, 44(3), 297-337.

Geva, S., Jones, P. S., Crinion, J. T., Price, C. J., Baron, J. C., \& Warburton, E. A. (2011). The neural correlates of inner speech defined by voxel-based lesion-symptom mapping. Brain, 134, 3071-3082.

Goschke, T. (2000). Intentional reconfiguration and involuntary persistence in task set switching. In S. Monsell \& J. Driver (Eds.), Control of cognitive processes: Attention and performance. (Vol. XVIII, pp. 331-355). Cambridge, MA: MIT Press. 
Jou, S.-C., Schultz, T., Walliczek, M., Kraft, F., \& Waibel, A. (2006). Towards continuous speech recognition using surface electromyography. Interspeech, 573-576.

Kieffaber, P. D., Kruschke, J. K., Cho, R. Y., Walker, P. M., \& Hetrick, W. P. (2013). Dissociating stimulus-set and response-set in the context of task-set switching.t Journal Of Experimental Psychology: Human Perception And Performance, 39(3), 700-719.

Kirkham, A. J., Breeze, J. M., \& Marí-Beffa, P. (2012). The impact of verbal instructions on goal-directed behaviour. Acta Psychologica, 139, 212-219.

Kray, J., Eber, J., \& Karbach, J. (2008). Verbal self-instructions in task switching: A compensatory tool for action-control deficits in childhood and old age? Developmental Science, 11(2), 223-236.

Lien, M. C., Ruthruff, E., \& Kuhns, D. (2008). Age-related differences in switching between cognitive tasks: Does internal control ability decline with age? Psychology and Aging, $23,330-341$.

Lupyan, G. (2009). Extracommunicative functions of language: Verbal interference causes selective categorization impairments. Psychonomic Bulletin \& Review, 16(4), 711718.

Luria, A. R. (1969). Speech development and the formation of mental processes. In M. Cole \& I. Maltzman (Eds.), A handbook of contemporary soviet psychology. New York, NY: Basic Books.

Mayr, U., Kleffner, K., Kikumoto, A., \& Redford, M. (2014). Control of Task Sequences: What is the Role of Language? Journal of Experimental Psychology: Learning, Memory, and Cognition, 40, 376-384.

Meiran, N. (2000). Modeling cognitive control in task-switching. Psychological Research, 63(3-4), 234-249. 
Miyake, A., Emerson, M. J., Padilla, F., \& Ahn, J.-C. (2004). Inner speech as a retrieval aid for task goals: The effects of cue type and articulatory suppression in the random task cuing paradigm. Acta Psychologica, 115(2-3), 123-142.

Monsell, S. (2003). Task switching. Trends in Cognitive Sciences, 7, 134-140.

Perrone-Bertolotti, M. M., Rapin, L. L., Lachaux, J. P., Baciu, M. M., \& Lœvenbruck, H. H. (2014). What is that little voice inside my head? Inner speech phenomenology, its role in cognitive performance, and its relation to self-monitoring. Behavioural Brain Research, 261, 220-239.

Reed, H. B. (1916). The existence and function of inner speech in thought processes. Journal of Experimental Psychology, 5, 365-392.

Saeki, E., \& Saito, S. (2004). Effect of articulatory suppression on task-switching performance: Implications for models of working memory. Memory, 12(3), 257-271.

Sokolov, A. N. (1972). Inner Speech and Thought. New York, NY: A Plenum / Rosetta edition. 